\title{
Novel Chimerical Endolysins with Broad Antimicrobial Activity Against Methicillin-Resistant Staphylococcus aureus
}

\author{
Sofia Fernandes,, ${ }^{1,2}$ Daniela Proença, ${ }^{1, *}$ Cátia Cantante, ${ }^{1, *}$ Filipa Antunes Silva, \\ Clara Leandro, Sara Lourenço, Catarina Milheiriço, ${ }^{3}$ Hermínia de Lencastre, ${ }^{3,4}$ \\ Patrícia Cavaco-Silva, ${ }^{1,5}$ Madalena Pimentel, ${ }^{2}$ and Carlos São-José ${ }^{2,6}$
}

Due to their bacterial lytic action, bacteriophage endolysins have recently gained great attention as a potential alternative to antibiotics in the combat of Gram-positive pathogenic bacteria, particularly those displaying multidrug resistance. However, large-scale production and purification of endolysins is frequently impaired due to their low solubility. In addition, a large number of endolysins appear to exhibit reduced lytic efficacy when compared with their action during phage infection. Here, we took advantage of the high solubility of two recently characterized enterococcal endolysins to construct chimeras targeting Staphylococcus aureus. The putative cell wall binding domain of these endolysins was substituted by that of a staphylococcal endolysin that showed poor solubility. Under appropriate conditions the resulting chimeras presented the high solubility of the parental enterococcal endolysins. In addition, they proved to be broadly active against a collection of the most relevant methicillin-resistant $S$. aureus epidemic clones and against other Gram-positive pathogens. Thus, fusion of endolysin domains of heterologous origin seems to be a suitable approach to design new potent endolysins with changed and/or extended lytic spectrum that are amenable to large-scale production.

\section{Introduction}

A FTER DECADES OF ALMOST exclusive use of antibiotics in the treatment of infectious diseases caused by pathogenic bacteria, the emergence of multiresistant bacterial strains combined with a slowdown in the discovery of new classes of antibiotics is currently viewed as a major public health problem. ${ }^{23,78,79}$ Among the different bacterial pathogens that have acquired antibiotic resistance Staphylococcus aureus is still one of the most serious threats worldwide, being able to develop resistance to virtually all classes of antibiotics. ${ }^{19}$ Methicillin-resistant S. aureus (MRSA) has been a leading cause of nosocomial infections for some decades and more recently has also emerged as a community-associated pathogen. $5,12,30,40$ S. aureus is responsible for a wide number of infectious diseases ranging from skin and soft tissues to fatal bloodstream infections, endocarditis, meningitis, and bovine mastitis in dairy herds. ${ }^{34,45}$ For all these reasons, there is an urgent need to develop new antibacterials that ensure elimination of multidrug-resistant pathogenic bacteria, particularly MRSA strains.

Among the different approaches to obtain new antibacterials, one that has recently caught great attention is the exploitation of bacteriophage endolysins. ${ }^{27,29,48,59}$ Endolysins are bacterial cell wall-hydrolyzing enzymes that promote host cell lysis in the end of the lytic cycle of double-stranded DNA phages, thus allowing efficient release of the viral progeny to the extracellular medium. ${ }^{71}$ Despite the fact that endolysins have been evolutionarily designed to operate from the inside of infected cells, when exogenously applied as purified recombinant proteins they have been shown to promote lysis of different Gram-positive pathogenic bacteria, including $S$. aureus. ${ }^{47,55,58,80}$

\footnotetext{
${ }^{1}$ Technophage, SA, Lisboa, Portugal.

${ }^{2}$ Centro de Patogénese Molecular, Unidade de Retrovírus e Infecções Associadas (CPM-URIA), Faculdade de Farmácia da Universidade de Lisboa, Lisboa, Portugal.

${ }^{3}$ Laboratory of Molecular Genetics, Instituto de Tecnologia Química e Biológica (ITQB), Universidade Nova de Lisboa, Oeiras, Portugal.

${ }^{4}$ Laboratory of Microbiology, The Rockefeller University, New York, New York.

${ }^{5}$ Center for Interdisciplinary Research Egas Moniz, Monte de Caparica, Portugal.

${ }^{6}$ Institute of Molecular Medicine, Lisboa, Portugal.

*These authors contributed equally to the work.
} 
Most endolysins have a modular organization with a conserved N-terminal catalytic domain (CD) and a more diverse C-terminal cell wall binding domain (CWBD). ${ }^{28,32}$ However, several $S$. aureus phages produce endolysins with two CDs in their N-terminus, such as those from phages $\mathrm{K}$, $\phi 11$ and $\phi$ MR11. These lytic enzymes present a CHAP (cysteine, histidine-dependent amidohydrolases/peptidases) domain followed by an Amidase- 2 domain ( $N$-acetylmuramoyl-L-alanine amidase), where the CHAP seems to be the most effective in inducing lysis. ${ }^{54,58,63}$ Another feature commonly reported for $S$. aureus phage endolysins is their poor solubility when overexpressed in Escherichia coli, frequently leading to low yields of soluble protein. ${ }^{9,31,51,58}$

We have experienced this insolubility problem when attempting to purify a broad-host-range anti-staphylococcal endolysin available in our lab, Lys $87 .{ }^{11}$ Here, we describe a new strategy to overcome this problem that consisted of the construction of new chimerical endolysins composed of the Lys87 CWBD fused to the highly soluble CDs of two Enterococcus faecalis phage endolysins, Lys168 and Lys170. ${ }^{62}$ By employing this strategy, complemented with optimized expression conditions, we obtained two highly soluble lytic enzymes that are broadly active against a large cohort of $S$. aureus strains, including MRSA, and against other staphylococcal, streptococcal, and enterococcal species. To our knowledge, this corresponds to the first report of chimeras comprising domains of $S$. aureus and E. faecalis phage endolysins.

\section{Materials and Methods}

\section{Bacteria, culture media, and growth conditions}

The E. coli cloning strain XL1-Blue MRF' and its derivatives were grown at $37^{\circ} \mathrm{C}$ with aeration in Luria-Bertani (LB) medium. ${ }^{68}$ The E. coli expression strain CG61 ${ }^{72}$ and its derivatives were grown in LB in the same conditions, except that incubation temperature was $28^{\circ} \mathrm{C}$ before induction of protein production and $16^{\circ} \mathrm{C}$ afterward. When appropriate, LB medium was supplemented with kanamycin $(40 \mu \mathrm{g} / \mathrm{ml})$ and/or ampicillin $(100 \mu \mathrm{g} / \mathrm{ml})$ for plasmid selection. Lytic action of chimerical endolysins was assayed in 200 bacterial clinical isolates (Table 1, Supplementary Tables S1 and S4; Supplementary Data are available online at www.lie bertonline.com/mdr). Table 1 corresponds to a panel of 30 MRSA and 13 Methicillin-sensitive S. aureus (MSSA) typed strains. Table S1 lists $100 \mathrm{~S}$. aureus isolates from Technophage's collection, 42 MRSA, 29 MSSA, and 29 with unknown methicillin susceptibility, which were obtained from different Portuguese community and hospital settings between 2005 and 2008. Recombinant lytic enzymes were also tested in clinical isolates of other species, namely against Staphylococcus epidermidis $(n=10)$, Staphylococcus haemolyticus $(n=9)$, Staphylococcus saprophyticus $(n=7)$, Enterococcus faecalis $(n=12)$, Enterococcus faecium $(n=10)$, and Streptococcus pyogenes $(n=9)$ (Technophage collection, Table S4). The growth media for these bacteria were purchased from Biokar Diagnostics, Beauvais, France. Staphylococcus and Enterococcus species were cultured in Brain-Heart Infusion and Streptococcus pyogenes in Todd Hewitt broth supplemented with $2 \%$ of yeast extract (THY). Bacteria were grown at $37^{\circ} \mathrm{C}$, with aeration, except for Enterococcus that was grown without agitation. When necessary, $1.5 \%$ or $0.7 \%$ agar was added to these culture media to obtain solid or soft-agar plates, respectively. E. faecalis and S. aureus phages were propagated and purified by standard methods, ${ }^{15,42}$ either in soft-agar media or liquid broth supplemented with $\mathrm{CaCl}_{2}$ and $\mathrm{MgCl}_{2}$ (5 mM each).

\section{Identification and bioinformatics analysis of phage endolysins}

Genomic DNA from E. faecalis and S. aureus phages was extracted from CsCl-purified lysates ${ }^{77}$ and their complete nucleotide sequence determined (service purchased to Macrogen). DNA homology searches were carried out with BLASTN, ${ }^{81}$ using the NCBI non-redundant nucleotide sequences database. Recognition of phage genome putative genes was performed by integrating the results obtained with GeneMark.hmm and MetaGeneAnnotator web software. ${ }^{10,56}$ Endolysins Lys168 and Lys170 from E. faecalis phages F168/08 and F170/08, respectively, were characterized previously. ${ }^{62}$ Identification of $S$. aureus phage F87s/06 endolysin gene was based on BLASTP homology searches ${ }^{4}$ against the NCBI non-redundant protein sequences database, using the deduced gene product, and on prediction of protein functional domains using NCBI's CDD ${ }^{52}$ and Pfam (http://pfam.janelia.org/search). Assignment of putative linkers connecting protein functional domains was performed with SVM $^{25}$ and the SVM-joint output. Multiple protein sequence alignments were performed with ClustalW2. ${ }^{45}$

\section{Construction and cloning of lys168-87 and lys170-87 chimerical genes}

The coding sequences of the N-terminal regions of the enterococcal phage endolysins Lys168 and Lys170, which included CHAP and Amidase-2 CDs, respectively ${ }^{62}$ (Fig. 1A and Supplementary Fig. S1A), were polymerase chain reaction (PCR) amplified from the corresponding phage DNA using a high-fidelity Pfu DNA Polymerase (Fermentas Molecular Biology Tools, Thermo Scientific). The sequence encoding the C-terminal region of the staphylococcal phage endolysin Lys87 (Fig. 1A and Supplementary Fig. S1A), and that harbored the putative CWBD, was similarly amplified by PCR in a separate reaction. The forward primers used for CD amplification carried an $\mathrm{NcoI}$ restriction site in their $5^{\prime}$ end, whereas the reverse primer employed in CWBD amplification contained an $\mathrm{X} m a \mathrm{I}$ site. The reverse and forward primers used in CD and CWBD amplification, respectively, carried a 26-bp complementary segment in their $5^{\prime}$ end. This allowed fusing the coding sequence of each enterococcal endolysin CD to that of the CWBD of Lys87 by the technique of overlap-extension by polymerase chain reaction (OEPCR), ${ }^{37}$ using the CD forward and CWBD reverse primers and a mixture of the initial PCR products as template. The resulting PCR products, corresponding to the chimerical genes lys168-87 and lys170-87 (Fig. 1B and Supplementary Fig. S1B), were purified using the High Pure PCR Product Purification Kit (Roche Applied Science), double-digested with $\mathrm{NcoI}$ and $\mathrm{XmaI}$, and ligated to the equally digested pIVEX2.3d expression vector (Roche Applied Science). This vector is designed to drive the expression of cloned genes under the control of the phage T7 $\phi 10$ promoter and to allow the production of the corresponding proteins C-terminally fused to a hexahistidine tag. The E. coli strain XL1-Blue MRF' 
was transformed with these ligations as described previously. ${ }^{14}$ Transformants were selected in the presence of $100 \mu \mathrm{g} / \mathrm{ml}$ ampicillin and screened for the presence of the desired recombinant plasmids by PCR, using insert and vector complementary primers. Plasmid DNA from positive clones was extracted (Pure Link Quick Plasmid Miniprep Kit; Invitrogen) and the correct DNA structure confirmed by endonuclease restriction and DNA sequencing (Macrogen). The constructs pSF168-87 and pSF170-87 are pIVEX2.3d derivatives carrying lys168-87 and lys170-87, respectively.

\section{Production and purification of the chimerical endolysins Lys168-87 and Lys170-87}

E. coli strain CG61, which overproduces phage T7 RNA polymerase upon temperate upshift ${ }^{72}$ was transformed with plasmids pSF168-87 and pSF170-87 and transformants selected at $28^{\circ} \mathrm{C}$ in presence of $100 \mu \mathrm{g} / \mathrm{ml}$ ampicillin and $40 \mu \mathrm{g} /$ $\mathrm{ml}$ kanamycin. Production of soluble and active Lys168-87 and Lys170-87 by CG61 transformants was first confirmed by growing them over a dense lawn of autoclavated staphylococcal cells, incorporated in soft-agar LB medium, and checked for the presence of lysis halos around E. coli colonies (Supplementary Fig. S2).

Selected clones of each chimera were grown at $28^{\circ} \mathrm{C}$ until an optical density at $600 \mathrm{~nm}\left(\mathrm{OD}_{600}\right)$ of $0.8-1.0$, after which protein production was induced by changing cultures to a shaking water bath set to $42^{\circ} \mathrm{C}$. After $30 \mathrm{~min}$, cultures were transferred to an incubator at $16^{\circ} \mathrm{C}$ and agitated for an additional period of $14 \mathrm{hr}$. Cells from induced cultures were pelleted by centrifugation $\left(8,000 \mathrm{~g}, 30 \mathrm{~min}, 4^{\circ} \mathrm{C}\right)$ and resuspended in 1/50 volume of lysis buffer ( $20 \mathrm{mM}$ Hepes, $500 \mathrm{mM} \mathrm{NaCl}$, $20 \mathrm{mM}$ imidazole, $1 \%$ glycerol, and $1 \mathrm{mM}$ DTT [pH 6.5]) supplemented with $1 \times$ Complete Mini EDTA-free Protease Inhibitor Cocktail (Roche Applied Science). Cells were kept on ice and disrupted by sonication (Vibra Cell MS2T, Sonic Materials) by performing about 10 bursts of $1 \mathrm{~min}$ (amplitude 50, pulse 3, 30-40 W) intercalated with pauses of $1 \mathrm{~min}$. Insoluble material was sedimented by centrifugation $(20,000 \mathrm{~g}, 30 \mathrm{~min}$, $4^{\circ} \mathrm{C}$ ). The supernatant corresponding to the total soluble extract was filtered through $0.22 \mu \mathrm{m}$ and the chimerical enzymes purified by affinity chromatography using HisTrap ${ }^{\mathrm{TM}} \mathrm{HP}$ columns (GE Healthcare) coupled to an AKTA-Prime system (GE Healthcare). The column and elution buffers had the same composition of the lysis buffer, except that the imidazole concentration in the elution buffer was $500 \mathrm{mM}$. Eluted fractions were analyzed by sodium dodecyl sulfate polyacrylanude gel electrophoresis and Coomassie blue staining. ${ }^{44}$ Chimeras pure fractions were pooled, concentrated, and changed to an imidazole-free, phosphate-based endolysin buffer ( $50 \mathrm{mM}$ phosphate-Na, $500 \mathrm{mM} \mathrm{NaCl}, 25 \%$ glycerol, and $1 \mathrm{mM}$ DTT [pH 6.5]) using HiTrap ${ }^{\mathrm{TM}}$ Desalting columns (GE Healthcare). Protein concentrations were determined by the Bradford method (Bio-Rad Laboratories) using bovine serum albumin as standard. The enzymes were divided into small aliquots and kept at $-20^{\circ} \mathrm{C}$.

\section{Evaluation of the lytic action of chimerical endolysins against bacterial pathogens}

The ability of chimeras Lys168-87 and Lys170-87 to induce lysis of clinical strains from different bacterial species was evaluated by two different assays. When tested against a large number of bacterial isolates, these were individually cultured as indicated above until an $\mathrm{OD}_{600}$ of 0.8-1.0. Cells were recovered by centrifugation and resuspended in 1/100 volume of fresh medium. A $100 \mu \mathrm{l}$ sample of these cellular suspensions was diluted in $10 \mathrm{ml}$ of incorporation buffer ( $25 \mathrm{mM}$ phosphate- $\mathrm{Na}$ and $250 \mathrm{mM} \mathrm{NaCl}$ [pH6.5]) supplemented with $0.7 \%$ agar and poured in a Petri dish. Four quantities of the purified chimeras $(10,5,1$, and $0.2 \mu \mathrm{g}$, in $10 \mu$ final volume) were spotted on each bacterial lawn and after overnight incubation at $37^{\circ} \mathrm{C}$ checked for the presence of lysis halos. These were evaluated and scored $(-$ to +++$)$ according to their relative diameter and transparency.

Bacterial cell lysis was also studied in liquid medium. Selected strains were grown until an $\mathrm{OD}_{600}$ of $0.3-0.4$, centrifuged, and cells recovered in 1/2 volumes of a phosphate buffer $(25 \mathrm{mM}$ phosphate- $\mathrm{Na}$ and $250 \mathrm{mM} \mathrm{NaCl}[\mathrm{pH}$ 6.5]). One-milliliter cell suspensions were challenged with $10 \mu \mathrm{g} / \mathrm{ml}$ of each chimera separately or with a mixture of both enzymes, each at $5 \mu \mathrm{g} / \mathrm{ml}$. The $\mathrm{OD}_{600}$ variation was followed over time. At the end of each assay the surviving colony forming units (CFU)/ml were determined. Negative controls were equally prepared except that endolysin buffer was added instead of endolysin.

\section{Results \\ Production of chimerical endolysins targeting S. aureus}

We have recently characterized three phage endolysins, two from E. faecalis phages F170/08 and F168/08 and another from $S$. aureus phage F87s/06. ${ }^{62,11}$ In silico analysis of the endolysin from phage F170/08, that is, Lys170 identified a $\mathrm{CD}$ of the Amidase- 2 family in its amino-terminal region (Fig. 1A and Supplementary Fig. S1A). This family includes zinc amidases that have N-acetylmuramoyl-L-alanine amidase activity. ${ }^{13}$ The same analysis performed with endolysins Lys168 and Lys87 from phages F168/08 and F87s/ 06, respectively, revealed a CD of the CHAP family, 7,46,64 also located in the enzymes' N-terminal region (Fig. $1 \mathrm{~A}$ and Supplementary Fig. S1A). Enzymes from this family have been shown to cleave different amide bonds in the peptidoglycan mesh, frequently displaying amidase or endopeptidase activity.

Lys170 and Lys168 could be easily produced in E. coli in its soluble form, whereas Lys87 showed a high propensity to precipitate as inclusion bodies in all tested conditions. ${ }^{11,62}$ The enterococcal endolysins exhibited a narrow lytic spectrum with their activity being basically restricted to $E$. faecalis cells. $^{62}$ This feature might result from CWBD binding to a specific epitope present on the cell wall of E. faecalis cells and/or dependence of the CD on CWBD binding for activity. ${ }^{49}$ Lys170 showed better lytic performance when both enterococcal endolysins were tested against a large number of E. faecalis clinical strains. ${ }^{62}$

Having this in mind we have envisaged that replacing the CWBD of Lys170 and Lys168 by that of Lys87 would result in chimerical endolysins (Fig 1B and Supplementary Fig. $\mathrm{S} 1 \mathrm{~B})$ maintaining the high solubility properties of the parental enterococcal enzymes and the ability to recognize and act on S. aureus cells conferred by the parental Lys87 CWBD.

The coding sequences of the functional domains composing each chimerical endolysin were PCR-amplified, fused by 
A
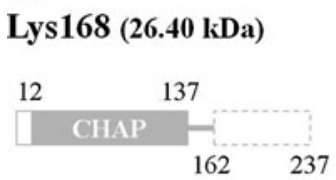

\section{Lys170 (31.37 kDa)}

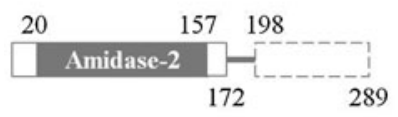

Lys170-87 (32.06 kDa)

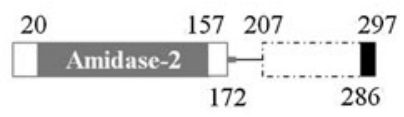

Lys87 (29.06) kDa

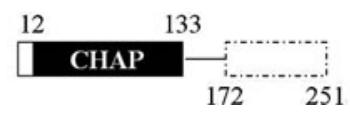

FIG. 1. Schematic representation of parental (A) and resulting chimerical endolysins (B). N-terminal catalytic domains (CD) are depicted as filled rectangles with indication of functional families (CHAP and Amidase-2). Dashed rectangles delimit the $\mathrm{C}$-terminal region that must contain the putative CWBD. Linker regions connecting CD and CWBD are indicated by a line with the filling code and thickness of parental endolysins. The black rectangle at the end of each chimera represents the C-terminal hexahistidine tag used for affinity chromatography purification. The amino acidic coordinates of functional and linker domains and the total length of each endolysin are indicated above and below the schemes. CWBD, cell wall binding domain; CHAP, cysteine, histidine-dependent amidohydrolases/peptidases.

OE-PCR, and cloned in the E. coli expression vector pIVEX2.3d, which allowed production of the chimeras C-terminally fused to a hexahistidine tag (Fig. 1B, Supplementary Fig. S1B, and Materials and Methods). The ability of the chimerical enzymes Lys168-87 and Lys170-87 to lyse $S$. aureus cells was initially confirmed by growing the resulting protein-expressing E. coli clones on a dense lawn of autoclavated staphylococcal cells and checking for the presence of lysis halos around the E. coli colonies (Supplementary Fig. S2).

By employing standard protein expression conditions $\left(37^{\circ} \mathrm{C}, 3-4 \mathrm{hr}\right.$ production after culture induction) with selected clones we could obtain much higher soluble amounts of the chimerical endolysins than those we had previously obtained with Lys87. However, the quantity of soluble Lys168-87 and Lys170-87 did not match the levels we achieved with the parental enterococcal endolysins, as we anticipated (not shown). This could be overcome though by simply incubating the induced cultures at $16^{\circ} \mathrm{C}$ during $14 \mathrm{hr}$. In these conditions we achieved similar or higher soluble quantities of the chimeras when compared with the native enterococcal enzymes. The chimeras were subsequently purified from total soluble extracts by affinity chromatography using nickel columns. Pure fractions were pooled and subjected to a desalting step for protein exchange to an imidazolefree, sodium phosphate-based buffer (Fig. 2). Average yields were $20 \mu \mathrm{g}$ and $30 \mu \mathrm{g}$ of pure Lys168-87 and Lys170-87, respectively, per $\mathrm{ml}$ of induced culture.

\section{Lytic action of the chimerical endolysins against clinical \\ S. aureus}

The lytic action of Lys168-87 and Lys170-87 against S. aureus was first assessed using a panel of 100 clinical isolates from Technophage's collection (Supplementary Table S1), which were isolated from different infection contexts and obtained both from Portuguese community and hospital settings. This panel included 42 isolates identified as MRSA. The lytic capacity of each chimera was evaluated by spotting four different amounts of pure enzyme $(10,5,1$, and $0.2 \mu \mathrm{g})$ on a dense lawn of viable cells from each tested isolate, which was produced by incorporating cells from exponentially growing cultures in a soft-agar, phosphate-buffered medium (see Materials and Methods). The chimera-induced lysis was qualitatively evaluated by scoring the relative diameter and turbidity of the lysis halos produced after overnight incubation at $37^{\circ} \mathrm{C}$ (Supplementary Table S2).

For the highest tested protein amount $(10 \mu \mathrm{g})$ we observed that both chimeras were able to produce lysis halos in more than $90 \%$ of the isolates (Supplementary Fig. S3). For each
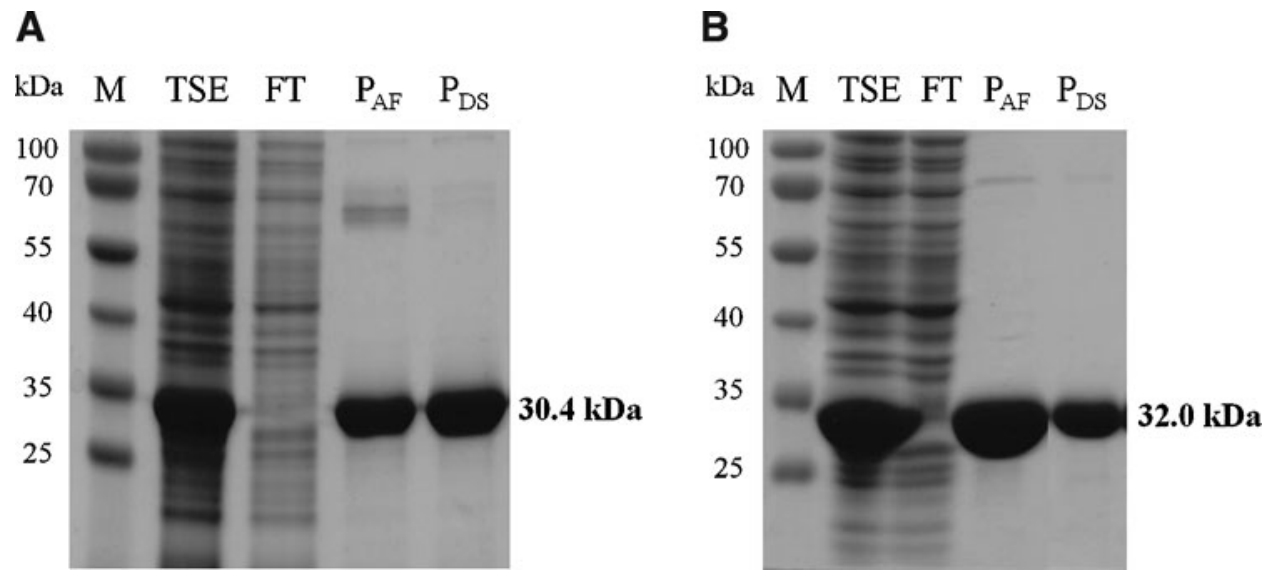

FIG. 2. Sodium dodecyl sulfate polyacrylamide gel electrophoresis (SDS-PAGE) analysis of Lys168-87 (A) and Lys170-87 (B) purification. M, molecular weight marker; TSE, total soluble extract loaded in the affinity column; FT, flowthrough of the affinity column; $\mathrm{P}_{\mathrm{AF}}$, eluted fraction of the affinity peak; $\mathrm{P}_{\mathrm{DS}}$, eluted fraction of the desalting peak. 
Table 1. Detailed Description of Typed Staphylococcus aureus Clinical Strains

\begin{tabular}{|c|c|c|c|c|c|c|c|}
\hline Strain ID & Origin & Isolation date & spa typing Ridom & $M L S T(S T)$ & $C C^{*}$ & SCCmec type & Reference \\
\hline \multicolumn{8}{|l|}{ MRSA, $n=30$} \\
\hline N315 & Japan & 1982 & t002 & 5 & 5 & II & 43,60 \\
\hline USA100 & United States & 1995-2003 & t002 & 5 & 5 & II & 53 \\
\hline JP1 & Japan & 1987 & t002 & 5 & 5 & II & 2,60 \\
\hline BM18 & United States & 1989 & t002 & 5 & 5 & IVa & 18,60 \\
\hline HDE288 & Portugal & 1996 & $\mathrm{t} 311$ & 5 & 5 & VI & 60,67 \\
\hline COB3 & Colombia & 1996 & $\mathrm{t} 1107$ & 5 & 5 & $\mathrm{IV}_{\mathrm{NT}}$ & 33,60 \\
\hline USA300 & United States & 1995-2003 & t008 & 8 & 5 & $\mathrm{IVa}$ & 53 \\
\hline GRE120 & Greece & 1993 & t036 & 8 & 5 & IVh & 1 \\
\hline JH1 & United States & 2000 & t002 & 105 & 5 & II & 75 \\
\hline JH9 & United States & 2000 & t002 & 105 & 5 & II & 75 \\
\hline ANS46 & Australia & 1982 & t037 & 239 & 5 & III & $24,36,60$ \\
\hline DEN907 & Denmark & 2001 & t037 & 239 & 5 & NT & 26,36 \\
\hline HDG2 & Portugal & 1992 & $\mathrm{t} 421$ & 239 & 5 & IIIB & 36,69 \\
\hline HPV107 & Portugal & 1992 & t051 & 247 & 5 & IA & 60,70 \\
\hline BK1953 & United States & 1995 & t051 & 247 & 5 & IA & 60,65 \\
\hline PER184 & Spain & 1991 & $\mathrm{t} 121$ & 247 & 5 & IA & 21,60 \\
\hline BK793 & Egypt & 1961 & t008 & 250 & 5 & $\mathrm{I}$ & 41,60 \\
\hline COL & United Kingdom & 1965 & t051 & 250 & 5 & I & 60 \\
\hline MW2 & United States & 1998 & $\mathrm{t} 128$ & 1 & 15 & $\mathrm{IVa}$ & 6 \\
\hline USA400 & United States & 1995-2003 & $\mathrm{t} 558$ & 1 & 15 & $\mathrm{IVa}$ & 53 \\
\hline HAR22 & Finland & 2002 & $\mathrm{t} 032$ & 22 & 22 & IVh & 35,66 \\
\hline HGSA146 & Portugal & 2003 & $\mathrm{t} 032$ & 22 & 22 & IVh & 1 \\
\hline DEN2294 & Denmark & 2001 & t019 & 30 & 30 & $\mathrm{IVC}_{\mathrm{C}}$ & 26 \\
\hline DEN2946 & Denmark & 2001 & $\mathrm{t} 975$ & 30 & 30 & IVc & 26 \\
\hline USA200 & United States & 1995-2003 & t018 & 36 & 30 & II & 53 \\
\hline HAR24 & Finland & 2002 & t018 & 36 & 30 & II & 35,66 \\
\hline HAR38 & Belgium & 1995 & t004 & 45 & 45 & IVa & 35,66 \\
\hline WIS & Australia & 1995 & $\mathrm{t} 123$ & 45 & 45 & V & 39 \\
\hline GRE14 & Greece & 1998 & t044 & 80 & 80 & IV & 1 \\
\hline DEN114 & Denmark & 2001 & t044 & 80 & 80 & IVc & 26 \\
\hline \multicolumn{8}{|l|}{ MSSA, $n=13$} \\
\hline HSA29 & Portugal & 1992-1993 & t002 & 5 & 5 & NA & 3 \\
\hline NCTC8325 & United Kingdom & 1943 & $\mathrm{t} 211$ & 8 & 5 & NA & 16,57 \\
\hline DCC403 & Portugal & 1996-1997 & $\mathrm{t} 148$ & 615 & 5 & NA & 3 \\
\hline Draftees728 & Portugal & 1996-1997 & $\mathrm{t} 213$ & 12 & 12 & NA & 3 \\
\hline IPOP33 & Portugal & 2001 & $\mathrm{t} 1071$ & 9 & 15 & NA & 3 \\
\hline DCC192 & Portugal & 1996-1997 & t084 & 15 & 15 & NA & 3 \\
\hline DCC750 & Portugal & 1996-1997 & t078 & 25 & 15 & NA & 3 \\
\hline IPOP58 & Portugal & 2001 & t189 & 188 & 15 & NA & 3 \\
\hline Draftees721 & Portugal & 1996-1997 & t790 & 22 & 22 & NA & 3 \\
\hline DCC1060 & Portugal & 1996-1997 & t012 & 30 & 30 & NA & 3 \\
\hline IPOP34 & Portugal & 2001 & t884 & 34 & 30 & NA & 3 \\
\hline DCC457 & Portugal & 1996-1997 & $\mathrm{t} 330$ & 45 & 45 & NA & 3 \\
\hline IPOP74 & Portugal & 2001 & t645 & 121 & 121 & NA & 3 \\
\hline
\end{tabular}

${ }^{*}$ Clonal complexes were determined using the E-burst software http://saureus.mist.net/, last accessed on January 25, 2012.

NA, not applied; NT, non-typeable; MLST (ST), multilocus sequence typing (sequence type); SCCmec, Staphylococcal Chromosomal Cassette mec; MRSA, methicillin-resistant S. aureus; MSSA, methicillin-sensitive S. aureus; CC, clonal complex.

protein quantity though, Lys168-87 generally produced larger and more transparent lysis halos than Lys170-87 (Supplementary Table S2). The narrowing of the lytic spectrum of this chimera as the spotted protein amount decreased was more pronounced than that observed with Lys168-87 (Supplementary Fig. S3). Interestingly, these results were basically opposite to those we obtained with the parental Lys168 and Lys170, where the latter exhibited better lytic performance against E. faecalis. ${ }^{62}$

Although both chimerical endolysins revealed to be very effective in lysing this panel of $S$. aureus clinical isolates $(n=100)$, these were not typed and therefore, the diversity present in this group was unknown. To have a more precise view on the lytic potential of the chimeras against $S$. aureus, the enzymes were similarly tested in a panel composed of diverse and typed MRSA and MSSA strains (Table 1). This strain collection was previously characterized in terms of their genetic background and included representatives of the most relevant MRSA pandemic clones from different parts of the world and representatives of the dominant of MSSA clones.

Remarkably, the chimerical endolysins lysed about 70\% to $100 \%$ of this panel of typed S. aureus clinical strains, depending on the amount of tested enzyme (Fig. 3). Lys168-87 again showed a better lytic performance when compared with Lys170-87 since $0.2 \mu \mathrm{g}$ of the former were 


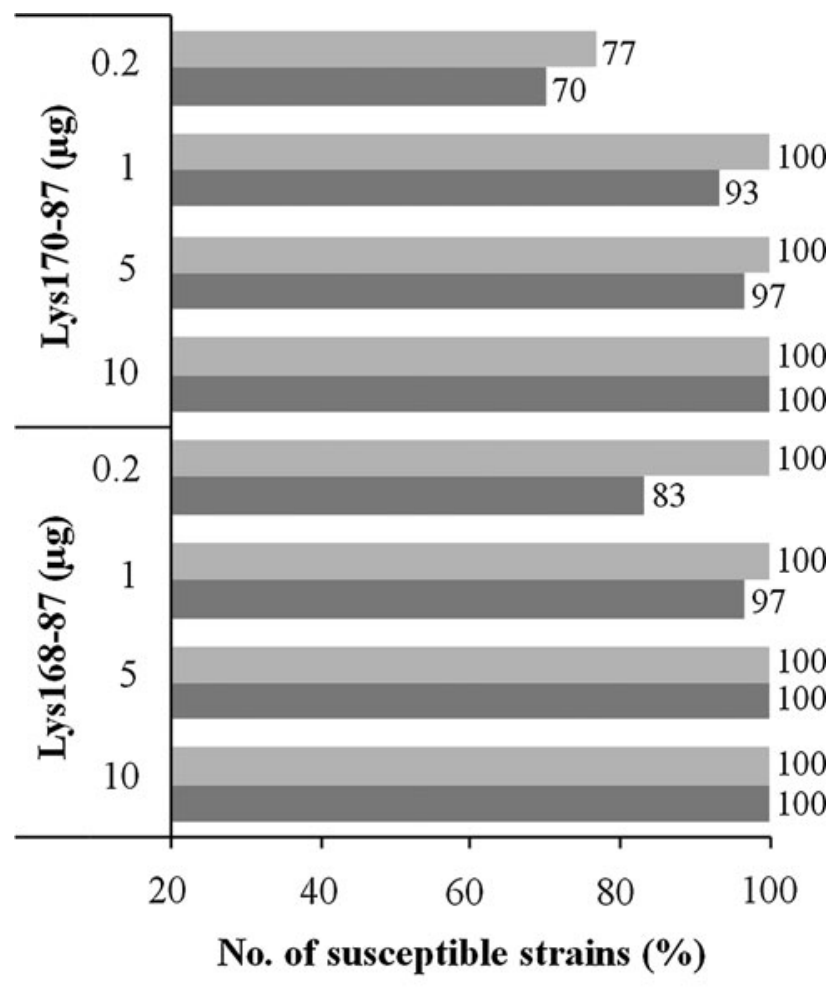

\section{$\operatorname{MSSA}(\mathrm{n}=13) \quad$ MRSA $(\mathrm{n}=30)$}

FIG. 3. Susceptibility of a panel of diverse and typed Staphylococcus aureus clinical strains to the lytic action of Lys16887 and Lys170-87. Typed MRSA and MSSA strains (Table 1) were challenged by the spot assay (see text) with the indicated amounts of each chimera. The percentage of strains that developed a lysis halo after overnight incubation at $37^{\circ} \mathrm{C}$ is plotted as a function of each enzyme amount. A detailed description of the susceptibility of each strain to both chimeras is presented in Supplementary Table S3. MRSA, Methicillin-resistant S. aureus; MSSA, Methicillin-sensitive S. aureus.

sufficient to produce a lysis halo in $83 \%$ of the tested MRSA strains, whereas Lys170-87 lysed only 70\%. A detailed description of the lytic activity of the endolysins in each particular $S$. aureus strain is shown in Supplementary Table S3.

\section{Lys168-87 and Lys170-87 have a synergistic lytic effect in liquid media}

The chimerical endolysins were also able to induce lysis of viable $S$. aureus cells in dense liquid suspensions, as shown in the example of Fig. 4, for the MRSA epidemic clone USA200. When applied separately, each endolysin caused a drop of cell suspension turbidity to about $40 \%$ of the initial value, after $1 \mathrm{hr}$ incubation, which translated into a corresponding decrease of the initial CFU/ml. However, if simultaneously added to cultures, each at half of the concentration when used separately, the chimerical enzymes decreased the cell suspension turbidity to almost $5 \%$ of the initial value and eliminated $\sim 99 \%$ of the initial CFU $/ \mathrm{ml}$ (Fig. 4). These results indicate a synergistic effect of both endolysins in the effective lysis and killing of $S$. aureus.

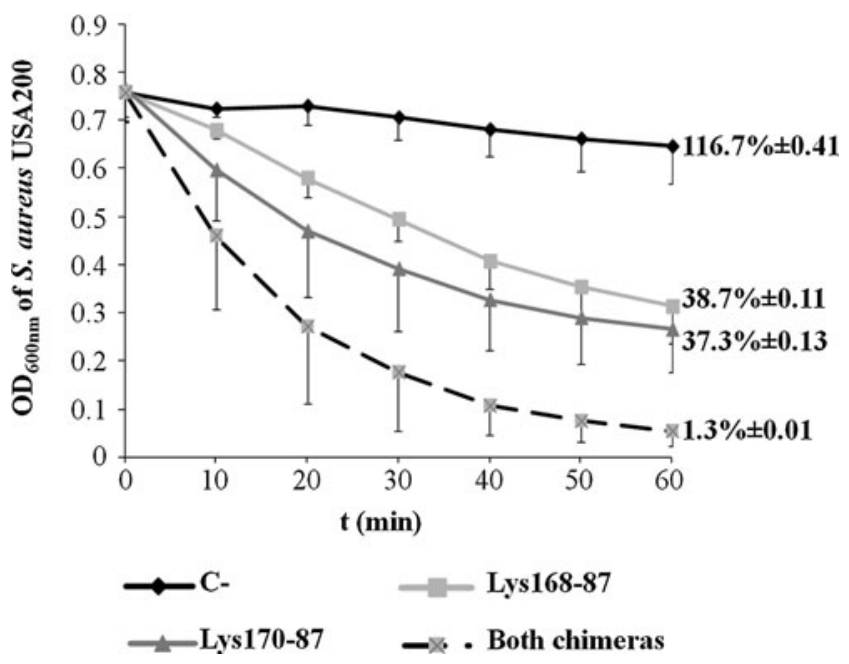

FIG. 4. Lytic action of Lys168-87 and Lys170-87 against S. aureus strain USA200 in liquid media. The graphic shows the change of cell suspensions $\mathrm{OD}_{600}$ over time, after adding each chimera separately $(10 \mu \mathrm{g} / \mathrm{ml})$ or in combination $(5 \mu \mathrm{g}$ / $\mathrm{ml}$ each). Endolysin buffer was added to the negative control $(\mathrm{C}-)$ instead of the enzymes. Values are the means of three independent experiments with indication of standard deviation. The values on the right side indicate the percentage of the initial CFU/ml after $60 \mathrm{~min}$ of enzymes' action and the corresponding standard deviation. OD, optical density.

\section{Activity of the chimerical endolysins against other Gram-positive pathogenic bacteria}

As mentioned before, the parental enterococcal endolysins Lys168 and Lys170 showed a clear preference for E. faecalis when tested against a representative group of typed strains of this species and of E. faecium. When comparing their lytic action, Lys170 showed to be more effective than Lys168. ${ }^{62}$ By replacing their putative CWBD by that of the staphylococcal endolysin Lys87 we could efficiently retarget their activity toward S. aureus, as initially envisaged (see above). Next, we studied how the chimeras acted on Gram-positive pathogenic bacteria other than S. aureus (Supplementary Table S4). When tested against the indicated E. faecalis and E. faecium clinical isolates, the chimerical enzymes essentially reproduced the lytic profile displayed by the parental enterococcal endolysins, ${ }^{62}$ with Lys170-87 producing larger and clearer lysis halos compared with Lys168-87 (Fig. 5 and Supplementary Table S5). Note that the E. faecalis and E. faecium isolates tested here were not genetically characterized and the vast majority of them have been previously shown to be susceptible to both Lys168 and Lys170. ${ }^{62}$

Interestingly, and in contrast to the parental enterococcal endolysins, the chimeras were also able to cause lysis of clinical isolates of other staphylococcal pathogens and Group A streptococci (Fig. 5A and Supplementary Table S5). Qualitatively, Lys168-87 demonstrated equal or superior lytic action than Lys170-87 against all tested bacteria, except for the enterococcal isolates, where Lys170-87 showed to be more powerful (Fig. 5B and Supplementary Table S5).

\section{Discussion}

A survey of the literature indicates that researchers frequently have to deal with the poor solubility of different 


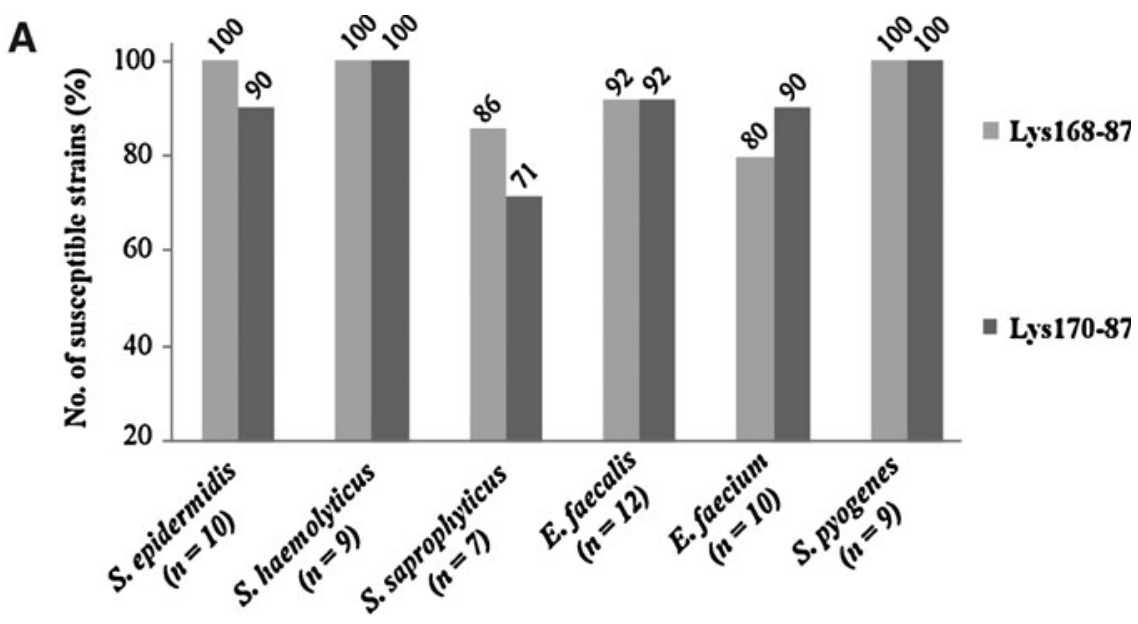

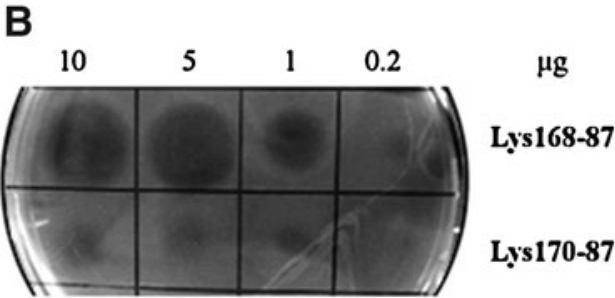

S. aureus 196/06

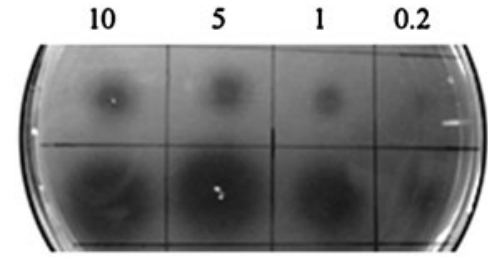

E. faecalis $926 / 05$

FIG. 5. Lytic action of Lys168-87 and Lys170-87 in other Gram-positive bacterial pathogens. (A) Bar plot showing the percentage of isolates from each species (Supplementary Table S4) that developed a lysis halo after spotting $10 \mu \mathrm{g}$ of each chimera. A detailed description of the susceptibility of each isolate to this and lower quantities of each chimera is presented in Supplementary Table S5. (B) Representative lysis halos for the indicated amounts of each chimera in a dense lawn of viable cells from S. aureus and E. faecalis isolates 196/06 and 926/05, respectively.

endolysins, including those of $S$. aureus phages, when aiming their production and purification. ${ }^{9,31,51,58,74,80}$ This is also a recurrent problem in our lab and generally the available technical approaches to increase solubility, or to refold proteins recovered from inclusions bodies, are time consuming and poorly effective.

We propose here that construction of chimeras, where we take advantage of the heterologous, highly soluble endolysin functional domains, may represent a solution to obtain large amounts of active lytic enzymes with retargeted and/or extended lytic spectrum relative to parental endolysins. As far as we know, this is the first time that such approach is employed with the specific objective of obtaining highly soluble lytic enzymes targeting $S$. aureus. There is though, at least one report where a similar strategy was attempted to solve the insolubility problem of an S. aureus phage endolysin. In this case the putative CD of the endolysin P16 from phage P68 was fused to the inferred CWBD of the virion-associated lysin P17, also from the same phage. However, the resulting chimera remained insoluble and accumulated as inclusion bodies. ${ }^{51}$

The construction of chimerical enzymes as a mean to improve the lytic efficacy or change the lytic spectrum, both in vitro and in vivo, toward relevant pathogens has been previously described. Recently, a chimerical endolysin (ClyS) assembling a CD and a CWBD from different $S$. aureus phages, and in which the latter displayed Staphylococcusspecific binding, was shown to lyse different $S$. aureus MRSA and MSSA strains and several coagulase-negative Staphylo- coccus species. ${ }^{17}$ This chimera revealed to be more effective than mupirocin for skin decolonization of MRSA and MSSA. ${ }^{61}$ In another example of homologous fusion, the CHAP domain of LysK, the endolysin from S. aureus phage $\mathrm{K}$, was fused to the CWBD of the bacteriocin lysostaphin from Staphylococcus simulans. ${ }^{38}$ The recombinant endolysin PRF-119 exhibited antimicrobial activity against a great number of MSSA and MRSA clinical isolates, with an average $\mathrm{MIC}_{90}$ of $0.391 \mu \mathrm{g} / \mathrm{ml}$.

Heterologous fusion constructs where the native CWBD of the streptococcal LambdaSa2 endolysin Cpl-7 was replaced by that of either lysostaphin or LysK resulted in a approximately fivefold increase in staphylolytic activity mediated by the Cpl-7 CD, while maintaining significant streptolytic activity. ${ }^{8}$ The results suggested that the CWBD of $S$. aureus lytic enzymes may not always be staphylococcal-specific. Yet in another example, chimeras composed of the streptococcal phage endolysin B30, or of its CHAP CD only, fused to the mature form of lysostaphin were shown to display lytic specificity for streptococcal pathogens and S. aureus. $^{22}$

In the work reported here, we have fused the CD of two highly soluble E. faecalis phage endolysins, Lys168 and Lys170, to the CWBD of the staphylococcal phage endolysin Lys87, which showed high propensity to precipitate in the form of inclusion bodies. Our rational was that the CD of the enterococcal enzymes would confer high solubility to the resulting chimerical endolysins. In addition, the fact that Lys168 and Lys170 CDs were of the CHAP and Amidase-2 
families, respectively, increased the chances of these domains to act on the $S$. aureus cell wall, if targeted by the Lys87 CWBD. Actually, the Lys168 CHAP domain, as deduced by Pfam analysis, shares $98 \%$ identity with that of the endolysin of S. aureus phage SAP6. ${ }^{62}$ Lys170 Amidase-2 CD should confer $\mathrm{N}$-acetylmuramoyl-L-alanine amidase activity as this was the activity experimentally determined for ORF9, an endolysin that is virtually identical to Lys170. ${ }^{62,76}$ Amidases cleave the amide bond that links the N-acetyl muramic acid of glycan strands to the L-alanine residue of peptide stems. This bond and linked residues are common to the vast majority of bacterial cell wall peptidoglycans, including that of S. aureus. ${ }^{73}$

The chimeras thus constructed proved to efficiently lyse $S$. aureus clinical strains. When tested at their highest concentration each recombinant endolysin was able to induce lysis in more than $96 \%$ of 143 S. aureus clinical isolates. The lytic efficiency of each chimera varied among the different isolates, as judged by the relative size and transparency of the lysis halos (Supplementary Tables S2 and S3). Remarkably, all the 30 typed strains composing a panel representative of the most relevant MRSA epidemic clones (Table 1) showed to be susceptible to both chimerical enzymes. Very few anti-staphylococcal endolysins have been tested in such a representative group of MRSA typed strains.

Also interesting was the fact that these chimerical endolysins not only essentially maintained the lytic performance of the parental enterococcal endolysins when tested against Enterococcus, ${ }^{62}$ but were also extended in their lytic spectrum being active against other Staphylococcus species and Streptococcus pyogenes. This suggests that the Lys87 CWBD may target a bacterial cell wall epitope that is common to several Gram-positive bacterial species, while that of the parental enterococcal endolysins binds to a ligand predominantly found on enterococcal cell wall. This apparent wider target range of the CWBD of anti-staphylococcal lytic enzymes was also observed for lysostaphin and LysK, as mentioned above. Thus, Lys168-87 and Lys170-87 can be added to the small list of broadly active, phage-derived lytic enzymes. $8,22,80$

A curious difference was observed when we compared the lytic efficiency of Lys168 and Lys170 with that of the derived chimeras Lys168-87 and Lys170-87. When tested against their natural target, E. faecalis, Lys170 generally produced larger and more transparent lysis halos than Lys168. However, this scenario was inverted when Lys168-87 and Lys17087 were assayed against $S$. aureus (see illustrative example in Fig. 5B). This appears to indicate that the lytic performance of a given endolysin is not a mere function of the affinity and binding of the CWBD to the cell wall. In fact, other parameters such as the affinity of the CD to its substrate may influence lytic activity. ${ }^{49}$

Lys168-87 and Lys170-87 could also lyse liquid cell suspensions of viable $S$. aureus. In addition, they showed a clear synergistic effect when simultaneously added to cells (Fig. 4). To the best of our knowledge, such synergy between phagederived hydrolytic enzymes in the effective lysis and killing of $S$. aureus has never been reported. Several studies though have demonstrated the occurrence of synergy between $S$. aureus phage endolysins and bacteriocins such as nisin ${ }^{31}$ or lysostaphin ${ }^{9}$ or between endolysins and conventional antibiotics. $^{17,20,51,63}$
In conclusion, our results indicate that the engineering of chimerical endolysins from heterologous functional domains can be a good strategy to obtain large quantities of soluble and highly effective peptidoglycan hydrolases, either with narrow or broad lytic spectrum. The efficacy of the chimeras Lys168-87 and Lys170-87 is currently under study in animal models of $S$. aureus infection.

\section{Acknowledgments}

D. Proença and C. Cantante work has been supported through Ph.D. fellowships SFRH/BD/64177/2009 and SFRH/BD/48598/2008, respectively from Fundação para a Ciência e a Tecnologia (FCT, MCTES, Portugal). C. Milheiriço was supported by Postdoctoral fellowship SFRH/BPD/ 63992/2009 from FCT.

\section{Author Disclosure Statement}

Technophage has proprietary rights over E. faecalis phages F168/08, F170/08, and S. aureus phage F87s/06, their encoded endolysins Lys168, Lys170, and Lys87, and the derived chimerical enzymes Lys168-87 and Lys170-87. All authors reviewed and approved the present article before submission. All authors declare that there are no conflicts of interest. All authors contributed to the elaboration of the article. S.F., D.P. and C.C. were responsible for endolysin cloning, production, and purification; SF executed all experiments evaluating lytic action of chimerical endolysins; F.A.S., C.L., C.C., and S.L. were responsible for phage isolation, purification, and extraction of phage DNA; C.L. and S.L. performed phage genomic analysis; C.M. was responsible for handling and molecular typing of $S$. aureus typed clinical strains. H.deL., P.C.-S., M.G., M.P., and C.S.-J. were responsible for the experimental design and work supervision; C.S.-J. coordinated the collaborative work of the involved laboratories.

\section{References}

1. Aires de Sousa, M., and H. de Lencastre. 2003. Evolution of sporadic isolates of methicillin-resistant Staphylococcus aureus (MRSA) in hospitals and their similarities to isolates of community-acquired MRSA. J. Clin. Microbiol. 41:3806-3815.

2. Aires de Sousa, M., H. de Lencastre, I. Santos Sanches, K. Kikuchi, K. Totsuka, and A. Tomasz. 2000. Similarity of antibiotic resistance patterns and molecular typing properties of methicillin-resistant Staphylococcus aureus isolates widely spread in hospitals in New York City and in a hospital in Tokyo, Japan. Microb. Drug Resist. 6:253-258.

3. Aires de Sousa, M., T. Conceição, C. Simas, and H. de Lencastre. 2005. Comparison of genetic backgrounds of methicillin-resistant and -susceptible Staphylococcus aureus isolates from Portuguese hospitals and the community. J. Clin. Microbiol. 43:5150-5157.

4. Altschul, S.F., T.L. Madden, A.A. Schäffer, J. Zhang, Z. Zhang, W. Miller, and D.J. Lipman. 1997. Gapped BLAST and PSI-BLAST: a new generation of protein database search programs. Nucleic Acids Res. 25:3389-3402.

5. Annual report on the antimicrobial resistance surveillance in Europe. 2010. European Antimicrobial Resistance Surveillance Network (EARS-Net). Available at www.ecdc. europa.eu/en/publications/Publications/1111_SUR_AMR_ data.pdf.pdf (Online.) 
6. Baba, T., F. Takeuchi, M. Kuroda, H. Yuzawa, K. Aoki, A. Oguchi, Y. Nagai, N. Iwama, K. Asano, T. Naimi, H. Kuroda, L. Cui, K. Yamamoto, and K. Hiramatsu. 2002. Genome and virulence determinants of high virulence community-acquired MRSA. Lancet 359:1819-1827.

7. Bateman, A., and N.D. Rawlings. 2003. The CHAP domain: a large family of amidases including GSP amidase and peptidoglycan hydrolases. Trends Biochem. Sci. 28:234-237.

8. Becker, S.C., J. Foster-Frey, A.J. Stodola, D. Anacker, and D.M. Donovan. 2009. Differentially conserved staphylococcal SH3b_5 cell wall binding domains confer increased staphylolytic and streptolytic activity to a streptococcal prophage endolysin domain. Gene 443:32-41.

9. Becker, S.C., J. Foster-Frey, and D.M. Donovan. 2008. The phage K lytic enzyme LysK and lysostaphin act synergistically to kill MRSA. FEMS Microbiol. Lett. 287:185-191.

10. Besemer, J., and M. Borodovsky. 2005. GeneMark: web software for gene finding in prokaryotes, eukaryotes and viruses. Nucleic Acids Res. 33:W451-W454.

11. Cantante, C. 2008. Isolamento e caracterização de uma lisina de um bacteriofago que infecta Staphylococcus aureus [Master's thesis]. Faculty of Medicine, University of Lisbon, Lisbon.

12. Chambers, H.F. 2001. The changing epidemiology of Staphylococcus aureus? Emerg. Infect. Dis. 7:178-182.

13. Cheng, X., X. Zhang, J.W. Pflugrath, and F.W Studier. 1994. The structure of bacteriophage T7 lysozyme, a zinc amidase and an inhibitor of T7 RNA polymerase. Proc. Natl. Acad. Sci. U. S. A. 91:4034-4038.

14. Chung, C.T., S.L. Niemela, and R.H. Miller. 1989. One-step preparation of competent Escherichia coli: transformation and storage of bacterial cells in the same solution. Proc. Natl. Acad. Sci. USA 86:2172-2175.

15. Clokie, M., and A. Kropinski. 2009. Bacteriophages: Methods and Protocols, 1st edition. Humana Press, New York.

16. Conceição, T., M. Aires de Sousa, and H. de Lencastre. 2009. Staphylococcal interspersed repeat unit typing of Staphylococcus aureus: evaluation of a new multilocus variablenumber tandem-repeat analysis typing method. J. Clin. Microbiol. 47:1300-1308.

17. Daniel, A., C. Euler, M. Collin, P. Chahales, K.J. Gorelick, and V.A. Fischetti. 2010. Synergism between a novel chimeric lysin and oxacillin protects against infection by methicillin-resistant Staphylococcus aureus. Antimicrob. Agents Chemother. 54:1603-1612.

18. de Lencastre, H., A. de Lencastre, and A. Tomasz. 1996. Methicillin-resistant Staphylococcus aureus isolates recovered from a New York City hospital: analysis by molecular fingerprinting techniques. J. Clin. Microbiol. 34:2121-2124.

19. de Lencastre, H., D. Oliveira, and A. Tomasz. 2007. Antibiotic resistant Staphylococcus aureus: a paradigm of adaptive power. Curr. Opin. Microbiol. 10:428-435.

20. Djurkovic, S., J.M. Loeffler, and V.A. Fischetti. 2005. Synergistic killing of Streptococcus pneumoniae with the bacteriophage lytic enzyme Cpl-1 and penicillin or gentamicin depends on the level of penicillin resistance. Antimicrob. Agents Chemother. 49:1225-1228.

21. Dominguez, M.A., H. de Lencastre, J. Linares, and A. Tomasz. 1994. Spread and maintenance of a dominant methicillin-resistant Staphylococcus aureus (MRSA) clone during an outbreak of MRSA disease in a Spanish hospital. J. Clin. Microbiol. 32:2081-2087.

22. Donovan, D.M., S. Dong, W. Garrett, G.M. Rousseau, S. Moineau, and D.G. Pritchard. 2006. Peptidoglycan hydro- lase fusions maintain their parental specificities. Appl. Environ. Microbiol. 72:2988-2996.

23. Doyle, J.S., K.L. Buising, K.A. Thursky, L.J. Worth, and M.J. Richards. 2011. Epidemiology of infections acquired in intensive care units. Semin. Respir. Crit. Care Med. 32:115-138.

24. Dubin, D.T., S.G. Chikramane, B. Inglis, P.R. Matthews, and P.R. Stewart. 1992. Physical mapping of the mec region of an Australian methicillin-resistant Staphylococcus aureus lineage and a closely related American strain. J. Gen. Microbiol. 138:169-180.

25. Ebina, T., H. Toh, and Y. Kuroda. 2009. Loop-length dependent SVM prediction of domain linkers for highthroughput structural proteomics. Biopolymers 92:1-8.

26. Faria, N.A., D.C. Oliveira, H. Westh, D.L. Monnet, A.R. Larsen, R. Skov, and H. de Lencastre. 2005. Epidemiology of emerging methicillin-resistant Staphylococcus aureus (MRSA) in Denmark: a nationwide study in a country with low prevalence of MRSA infection. J. Clin. Microbiol. 43:1836-1842.

27. Fenton, M., P. Ross, O. McAuliffe, J. O'Mahony, and A. Coffey. 2010. Recombinant bacteriophage lysins as antibacterials. Bioeng. Bugs 1:9-16.

28. Fischetti, V.A. 2008. Bacteriophage lysins as effective antibacterials. Curr. Opin. Microbiol. 11:393-400.

29. Fischetti, V.A. 2010. Bacteriophage endolysins: a novel antiinfective to control Gram-positive pathogens. Int. J. Med. Microbiol. 300:357-362.

30. Furuya, E.Y., and F.D. Lowy. 2006. Antimicrobial-resistant bacteria in the community setting. Nat. Rev. Microbiol. 4: $36-45$.

31. García, P., B. Martínez, L. Rodríguez, and A. Rodríguez. 2010. Synergy between the phage endolysin LysH5 and nisin to kill Staphylococcus aureus in pasteurized milk. Int. J. Food Microbiol. 141:151-155.

32. Garcia, P., J.L. Garcia, E. Garcia, J.M. Sanchez-Puelles, and R. Lopez. 1990. Modular organization of the lytic enzymes of Streptococcus pneumonia and its bacteriophages. Gene 86:81-88.

33. Gomes, A.R., I.S. Sanches, M. Aires de Sousa, E. Castañeda, and H. de Lencastre. 2001. Molecular epidemiology of methicillin-resistant Staphylococcus aureus in 33. Colombian hospitals: dominance of a single unique multidrug-resistant clone. Microb. Drug Resist. 7:23-32.

34. Gruet, P., P. Maicent, X. Berthelo, and V. Kaltsatos. 2001. Bovine mastitis and intramammary drug delivery: review and perspectives. Adv. Drug Deliv. Rev. 50:245-259.

35. Harmony Project. HARMONY - the International Union of Microbiology Societies' European Staphylococcal Typing Network. Available at www.harmony-microbe.net. April 27, 2006. (Online.)

36. Harris, S.R., E.J. Feil, M.T. Holden, M.A. Quail, E.K. Nickerson, N. Chantratita, S. Gardete, A. Tavares, N. Day, J.A. Lindsay, J.D. Edgeworth, H. de Lencastre, J. Parkhill, S.J. Peacock, and S.D. Bentley. 2010. Evolution of MRSA during hospital transmission and intercontinental spread. Science 327:469-474.

37. Ho, S.N., H.D. Hunt, R.M. Horton, J.K. Pullen, and L.R. Pease. 1989. Site-directed mutagenesis by overlap extension using the polymerase chain reaction. Gene 77:51-59.

38. Idelevich, E.A., C. Von Eiff, A.W. Friedrich, D. Iannelli, G. Xia, G. Peters, A. Peschel, I. Wanninger, and K. Becker. 2011. In vitro activity against Staphylococcus aureus of a novel antimicrobial agent, PRF-119, a recombinant chimeric bacteriophage endolysin. Antimicrob. Agents Chemother. 55: 4416-4419. 
39. Ito, T., X.X. Ma, F. Takeuchi, K. Okuma, H. Yuzawa, and K. Hiramatsu. 2004. Novel type V staphylococcal cassette chromosome mec driven by a novel cassette chromosome recombinase, ccrC. Antimicrob. Agents Chemother. 48:26372651.

40. Klevens, R.M., M.A. Morrison, J. Nodle, S. Petit, K. Gershman, S. Ray, L.H. Harrison, R. Lynfield, G. Dumyati, J.M. Townes, A.S. Craig, E.R. Zell, et al. 2007. Invasive methicillin-resistant Staphylococcus aureus infections in the United States. JAMA 298:1763-1771.

41. Kreiswirth, B., J. Kornblum, R.D. Arbeit, W. Eisner, J.N. Maslow, A. McGeer, D.E. Low, and R.P. Novick. 1993. Evidence for a clonal origin of methicillin resistance in Staphylococcus aureus. Science 259:227-230.

42. Kutter, E., and A. Sulakvelidze. 2004. Bacteriophages: Biology and Applications, 1st edition. CRC Press, Boca Raton.

43. Kuwahara-Arai, K., N. Kondo, S. Hori, E. Tateda-Suzuki, and K. Hiramatsu. 1996. Suppression of methicillin resistance in a mecA-containing pre-methicillin-resistant Staphylococcus aureus strain is caused by the mecI-mediated repression of PBP $2^{\prime}$ production. Antimicrob. Agents Chemother. 40:2680-2685.

44. Laemmli, U.K. 1970. Cleavage of structural proteins during the assembly of the head of bacteriophage T4. Nature 227:680-685.

45. Larkin, M.A., G. Blackshields, N.P. Brown, R. Chenna, P.A. McGettigan, H. Mcwilliam, F. Valentin, I.M. Wallace, A. Wilm, R. Lopez, et al. 2007. ClustalW and ClustalX version 2. Bioinformatics 23:2947-2948.

46. Layec, S., B. Decaris, and N. Leblond-Bourget. 2008. Characterization of proteins belonging to the CHAP-related superfamily within the Firmicutes. J. Mol. Microbiol. Biotechnol. 14:31-40.

47. Loeffler, J.M., D. Nelson, and V.A. Fischetti. 2001. Rapid killing of Streptococcus pneumoniae with a bacteriophage cell wall hydrolase. Science 294:2170-2172.

48. Loessner, M.J. 2005. Bacteriophage endolysins-current state of research and applications. Curr. Opin. Microbiol. 8:480-487.

49. Low, L.Y., C. Yang, M. Perego, A. Osterman, and R. Liddington. 2011. Role of net charge on catalytic domain and influence of cell wall binding domain on bactericidal activity, specificity, and host range of phage lysins. J. Biol. Chem. 286:34391-34403.

50. Lowy, F.D. 1998. Staphylococcus aureus infections. N. Engl. J. Med. 339:520-532.

51. Manoharadas, S., A. Witte, and U. Bläsi. 2009. Antimicrobial activity of a chimeric enzybiotic towards Staphylococcus aureus. J. Biotechnol. 139:118-123.

52. Marchler-Bauer, A., J.B. Anderson, F. Chitsaz, M.K. Derbshire, C. DeWeese-Scott, J.H. Fong, L.Y. Geer, R.C. Geer, N.R. Gonzalez, et al. 2011. CDD: a Conserved Domain Database for the functional annotation of proteins. Nucleic Acids Res. 39:D225-D229.

53. McDougal, L.K., C.D. Steward, G.E. Killgore, J.M. Chaitram, S.K. McAllister, and F.C. Tenover. 2003. Pulsedfield gel electrophoresis typing of oxacillin-resistant Staphylococcus aureus isolates from the United States: establishing a national database. J. Clin. Microbiol. 41:51135120.

54. Navarre, W.W., H. Ton-That, K.F. Faull, and O. Schneewind. 1999. Multiple enzymatic activities of the murein hydrolase from staphylococcal phage $\Phi 11$. Identification of a
D-alanyl-glycine endopeptidase activity. J. Biol. Chem. 274:15847-15856.

55. Nelson, D., L. Loomis, and V.A. Fischetti. 2001. Prevention and elimination of upper respiratory colonization of mice by group A streptococci by using a bacteriophage lytic enzyme. Proc. Natl. Acad. Sci. U. S. A 98:4107-4112.

56. Noguchi, H., T. Taniguchi, and T. Itoh. 2008. MetaGeneAnnotator: detecting species-specific patterns of ribosomal binding site for precise gene prediction in anonymous prokaryotic and phage genomes. DNA Res. 15:387-396.

57. Novick, R. 1967. Properties of a cryptic high-frequency transducing phage in Staphylococcus aureus. Virology 33:155166.

58. O'Flaherty, S., A. Coffey, W. Meaney, G.F. Fitzgerald, and R.P. Ross. 2005. The recombinant phage lysin LysK has a broad spectrum of lytic activity against clinically relevant staphylococci, including methicillin-resistant Staphylococcus aureus. J. Bacteriol. 187:7161-7164.

59. O'Flaherty, S., R.P. Ross, and A. Coffey. 2009. Bacteriophage and their lysins for elimination of infectious bacteria. FEMS Microbiol. Rev. 33:801-819.

60. Oliveira, D.C., A. Tomasz, and H. de Lencastre. 2001. The evolution of pandemic clones of methicillin-resistant Staphylococcus aureus: identification of two ancestral genetic backgrounds and the associated mec elements. Microb. Drug Resist. 7:349-361.

61. Pastagia, M., C. Euler, P. Chahales, J. Fuentes-Duculan, J.G. Krueger, and V.A. Fischetti. 2011. A novel chimeric lysin shows superiority to mupirocin for skin decolonization of methicillin-resistant and -sensitive Staphylococcus aureus strains. Antimicrob. Agents Chemother. 55:738-744.

62. Proença, D., S. Fernandes, C. Leandro, F. Silva, S. Santos, M. Pimentel, F. Lopes, R. Mato, P. Cavaco-Silva, M. Garcia, and C. São-José. 2012. Phage endolysins with broad antimicrobial activity against Enterococcus faecalis clinical strains. Accompanying paper submitted to MDR, GWS-2011.

63. Rashel, M., J. Uchiyama, T. Ujihara, Y. Uehara, S. Kuramoto, and S. Sugihara. 2007. Efficient elimination of multidrugresistant Staphylococcus aureus by cloned lysin derived from bacteriophage $\phi$ MR11. J. Infect. Dis. 196:1237-1247.

64. Rigden, D.J., M.J. Jedrzejas, and M.Y. Galperin. 2003. Amidase domains from bacterial and phage autolysins define a family of gamma-D,L-glutamate-specific amidohydrolases. Trends Biochem. Sci. 28:230-234.

65. Roberts, R. B., A. de Lencastre, W. Eisner, E.P. Severina, B. Shopsin, B.N. Kreiswirth, and A. Tomasz. 1998. Molecular epidemiology of methicillin-resistant Staphylococcus aureus in 12 New York hospitals. MRSA Collaborative Study Group. J. Infect. Dis. 178:164-171.

66. Robinson, D.A., and M.C. Enright. 2003. Evolutionary models of the emergence of methicillin-resistant Staphylococcus aureus. Antimicrob. Agents Chemother. 47:3926-3934.

67. Sá-Leão, R., I. Santos Sanches, D. Dias, I. Peres, R.M. Barros, and H. de Lencastre. 1999. Detection of an archaic clone of Staphylococcus aureus with low-level resistance to methicillin in a pediatric hospital in Portugal and in international samples: relics of a formerly widely disseminated strain? J. Clin. Microbiol. 37:1913-1920.

68. Sambrook, J., and D.W. Russell. 2001. Molecular Cloning: A Laboratory Manual, 3rd edition. Cold Spring Harbor Laboratory Press, Cold Spring Harbor, NY.

69. Sanches, I.S., M. Aires de Sousa, L. Cleto, M.B. de Campos, and $\mathbf{H}$. de Lencastre. 1996. Tracing the origin of an outbreak of methicillin-resistant Staphylococcus aureus infections 
in a Portuguese hospital by molecular fingerprinting methods. Microb. Drug Resist. 2:319-329.

70. Sanches, I.S., M. Ramirez, H. Troni, M. Abecassis, M. Padua, A. Tomasz, and H. de Lencastre. 1995. Evidence for the geographic spread of a methicillin-resistant Staphylococcus aureus clone between Portugal and Spain. J. Clin. Microbiol. 33:1243-1246.

71. São-José, C., J. Nascimento, R. Parreira, and M.A. Santos. 2007. Release of progeny phages from infected cells. In S. Macgrath and D. van Sinderen (eds.), Bacteriophages: Genetics and Molecular Biology. Horizon Scientific Press and Caister Academic Press, Norwich, UK, pp. 309-336.

72. São-José, C., R. Parreira, G. Vieira, and M.A. Santos. 2000. The N-terminal region of the Oenococcus oeni bacteriophage fOg44 lysin behaves as a bona fide signal peptide in Escherichia coli and as a cis-inhibitory element, preventing lytic activity on oenococcal cells. J. Bacteriol. 182:5823-5831.

73. Schleifer, K.H., and O. Kandler. 1972. Peptidoglycan types of bacterial cell walls and their taxonomic implications. Bacteriol. Rev. 36:407-477.

74. Schmitz, J.E., M.C. Ossiprandi, K.R. Rumah, and V.A. Fischetti. 2011. Lytic enzyme discovery through multigenomic sequence analysis in Clostridium perfringens. Appl. Microbiol. Biotechnol. 89:1783-1795.

75. Sieradzki, K., T. Leski, J. Dick, L. Borio, and A. Tomasz. 2003. Evolution of a vancomycin-intermediate Staphylococcus aureus strain in vivo: multiple changes in the antibiotic resistance phenotypes of a single lineage of methicillinresistant $S$. aureus under the impact of antibiotics administered for chemotherapy. J. Clin. Microbiol. 41:1687-1693

76. Uchiyama, J., I. Takemura, I. Hayashi, S. Matsuzaki, M. Satoh, T. Ujihara, M. Murakami, M. Imajoh, M. Sugai, and M. Daibata. 2011. Characterization of lytic enzyme open reading frame 9 (ORF9) derived from Enterococcus faecalis bacteriophage $\phi E F 24 C$. Appl. Environ. Microbiol. 77:580585.

77. Vinga, I., C. Baptista, I. Auzat, I. Petipas, R. Lurz, P. Tavares, M.A. Santos, and C. São-José. 2012. Role of bacteriophage SPP1 tail spike protein gp21 on host cell receptor binding and trigger of phage DNA ejection. Mol. Microbiol. 83:289-303.

78. Willems, R.J., W.P. Hanage, D.E. Bessen, and E.J. Feil. 2011. Population biology of Gram-positive pathogens: highrisk clones for dissemination of antibiotic resistance. FEMS Microbiol. Rev. 35:872-900.

79. Woodford, N., J.F. Turton, and D.M. Livermore. 2011. Multiresistant Gram-negative bacteria: the role of high-risk clones in the dissemination of antibiotic resistance. FEMS Microbiol. Rev. 35:736-755.

80. Yoong, P., R. Schuch D. Nelson, and V.A. Fischetti. 2004. Identification of a broadly active phage lytic enzyme with lethal activity against antibiotic-resistant Enterococcus faecalis and Enterococcus faecium. J. Bacteriol. 186:4808-4812.

81. Zhang, Z., S. Schwartz, L. Wagner, and W. Miller. 2000. A greedy algorithm for aligning DNA sequences. J. Comput. Biol. 7:203-214.

Address correspondence to: Carlos São-José, Ph.D.

Dept de Microbiologia e Imunologia Faculdade de Farmácia da Universidade de Lisboa

Centro de Patogénese Molecular

Unidade de Retrovírus e Infecções Associadas (CPM-URIA)

Av. Prof. Gama Pinto 1649-003 Lisboa Portugal

E-mail: csaojose@ff.ul.pt 